\title{
CAD support and new developments in DD4hep
}

\author{
Markus Frank $^{1}$, Frank Gaede ${ }^{2}$, Marko Petrič ${ }^{1, *}$, and André Sailer ${ }^{1}$ \\ ${ }^{1}$ CERN, CH-1211 Geneva 23, Switzerland \\ ${ }^{2}$ DESY, Notkestraße 85, D-22607 Hamburg, Germany
}

\begin{abstract}
Consistent detector description is an integral part of all modern experiments and also the main motivation behind the creation of DD4hep, which tries to address detector description in a broad sense including for, example, geometry and the materials used in the device, additional parameters describing e.g. the detection techniques, constants required for alignment and calibration, description of the readout structures and conditions data. A central component of DD4hep is DDG4 which is a mechanism that converts arbitrary DD4hep detector geometries to Geant 4 and provides access to all Geant 4 action stages. In addition to that DDG4 also offers a comprehensive plugins suite that includes handling of different IO formats, Monte Carlo truth linking and a large set of segmentation and sensitive detector classes, allowing the simulation of a wide variety of detector technologies. One of the last remaining open issues of detector description was support for drawings from civil engineers for passive detector components. In this proceedings we highlight recent developments in DD4hep/DDG4 that enable support for CAD drawings and generic tessellated shapes and through the help of the library assimp enable the import of a wide variety of CAD formats, thus eliminating the need for writing complex re-implementations of CAD drawings in source code. In addition, we present other developments such as support for a new output format called EDM4hep and developments for a more unified and easier handling of units.
\end{abstract}

\section{Introduction}

The DD4hep software toolkit [1] targets the unification of detector description at all stages of the experimental lifecycle. The objective is to extract consistent information from a single source and deliver it to simulation, reconstruction and analysis steps in data processing. The advantages of such a consistent, comprehensive description are clearly established from experience in high energy physics experiments.

The design choices and foundations for DD4hep were based on experience from the LHCb experiment [2] and the efforts of the Linear Collider Community [3]. The aim is to deliver on one side the geometrical and material properties, and on the other side also provide parameters such as alignment constants and calibration, description of the readout structures and conditions, and those directly associated with detection techniques. The development of DD4hep was always guided by the desire to provide widely usable generic software to achieve a comprehensive detector description. The two main dependencies of DD4hep are

\footnotetext{
*e-mail: marko.petric@cern.ch
} 
ROOT, which is used for the construction and visualization of the detector geometry [4], and Geant4 which is used to perform detector simulation via an interface [5].

The working principles of DD4hep [6] and details about simulation [7] have been summarized at previous conferences [8]. The aim of these proceedings is to present Tessellated Shapes and CAD support as well as highlight the most relevant changes that occurred since release DD4hep v01-11, the current realease is v01-16.

\section{Main objectives}

The objectives which DD4hep aims to cover are:

- Complete detector description: A geometry description comprising all structures and the matching materials, the attributes necessary for visualization, the parameters needed for alignment, calibration, and environment description, as well as detector readout information.

- Coverage of the full life cycle of an experiment: Providing functionality for all stages in the life-cycle of the experiment, from concept development, through construction to operation, providing a smooth transition between various stages.

- Single source of information: Containing all of the required information for interpretation of data in all stages, from simulation to reconstruction and analysis.

- Ease of use: Exposes to the final user a simple and intuitive interface, which requires minimal external dependencies.

\section{The DD4hep toolkit}

At the core of DD4hep lies the Generic Detector Description Model (GDDM) which is built around the ROOT TGeo package. The GDDM is an in-memory model, it is comprised of a set of objects containing geometry and auxiliary information about the detector. Figure 1 shows a diagrammatic depiction of the various components of DD4hep, the interactions between them and the end-user applications, such as alignment, visualisation and reconstruction.

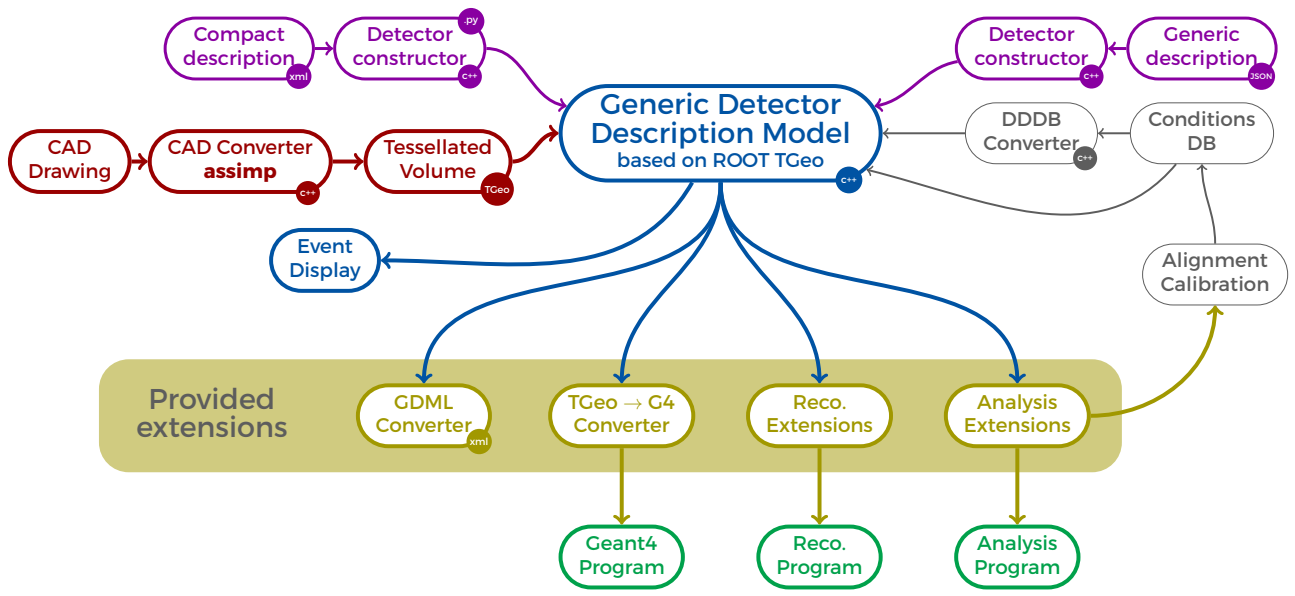

Figure 1. The components of the DD4hep detector geometry toolkit (in red new flow of the CAD interface). 


\section{Support for Tessellated Shapes and CAD Tools}

The support to create real parts of the detector geometry directly from design files provided by engineering departments using Computer Aided Design (CAD) tools was demanded by physicists for a long time. Such an approach obviously offers large benefits, firstly because no work is involved translating design drawings to the geometry description and secondly to minimize potential problems or errors when producing the geometry description. To achieve this goal two basic ingredients were necessary:

- The possibility to model arbitrary tessellated shapes as composites of tri- or quadrilinear facets both in the ROOT geometry description [4] and in Geant4 [5, 9].

- A generic mechanism to import arbitrary shapes described in CAD files to shapes understood by the ROOT geometry toolkit.

The Open Asset Import library (assimp) [10] is a portable Open-Source library to import export 3D-models using to a common abstraction layer consisting of tessellated shapes built by tri- or quadrilinear facets. For may formats the assimp library supports not only reading shapes from CAD files, but also the export of geometries to CAD files. When importing transient, assimp-specific tessellated shapes which are created from CAD files and then immediately translated to ROOT tessellated shapes.then immediately translated to ROOT tessellated shapes. When exporting ROOT based geometries, first transient, assimp-specific tessellated shapes are created, which then are written to file using the assimp library. The export mechanism allows to include detector geometries as predefined modules to CAD tools. Round-trip engineering to seemlessly integrate design activities and optimized simulation is supported with one caveat: once analytical shapes like boxes, tubes etc. are converted to tessellated shapes, it is not possible to convert them back to analytical shapes. Hence, import and export setups must use this feature very selectively.
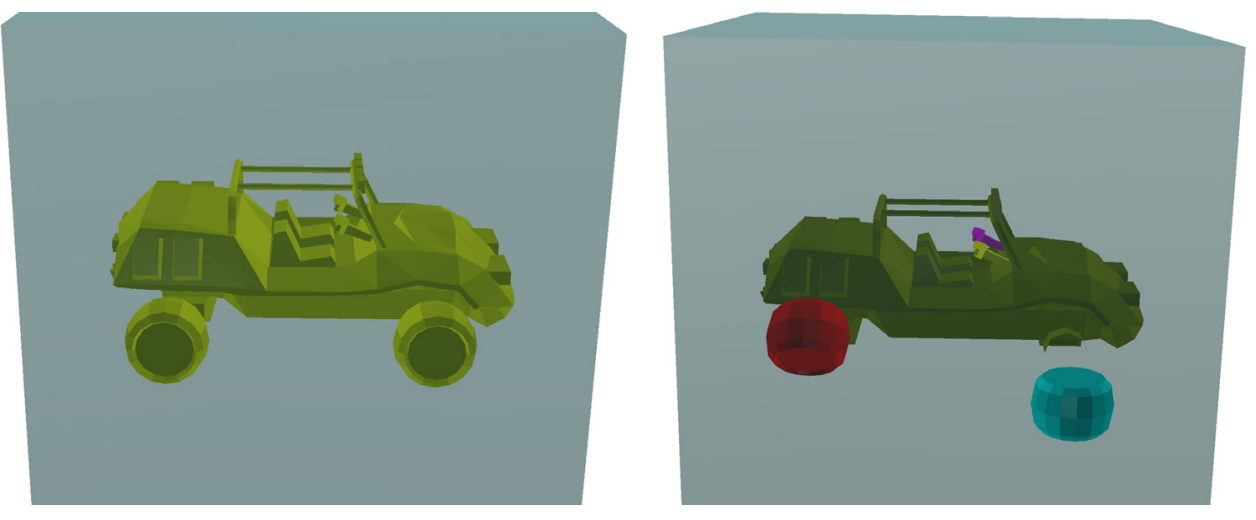

Figure 2. An example for a multi-mesh CAD input file converted to ROOT geometry. To the left the raw converted meshes with their natural placement are shown. To the right the same meshes are shown, but each mesh individually addressed by the plugin. Each mesh can be assigned its own material, visual attributes and corrected placement as supplied to the XML pattern.

Currently reading and writing CAD geometry files is offered to clients through the DD4hep plugin mechanism. The plugins are designed to be integrated in DD4hep detector constructor plugins [6]. Factories need to be called to invoke the required functionality. These mechanisms may be called either directly in the detector construction code [6] or can 
be triggered in XML snippets exposing certain patterns, which supply additional information. Future plans will center on the collaboration with clients to identify typical use cases. This should lead to a very convienient usage to instantiate tesselated shapes. The DDG4 [11] extension of DD4hep used to simulate detector geometries using the Geant4 [5] toolkit was extended to detect and automatically translate tessellated shapes to Geant4.

CAD files may contain not only one, but multiple meshes of facet based shapes as it is shown in Figure 2. Each of these meshes corresponds to a separate shape. To properly construct volumes used to build geometries each of these meshes/shapes can be addressed individually and can have its own material, visualization properties and positioning. It is only necessary to individually address sub-meshes if the used CAD file format does not support materials and visualization attributes. If materials and visualization attributes are supported by the CAD format, volumes are build directly using this information.

The Open Asset Import library supports a large variety of CAD formats which this way can be imported into the ROOT geometry system or exported from ROOT to CAD files. Roughly 50 different formats are supported [12] including popular specializations like OBJ, STL, STEP, X3D, DXF, FBX or Collada. Unfortunately the most accurate list of supported formats can onlty be deduced from the assimp code base. The choice of a format supporting the required functionality is important: not all formats support both model imports and exports. In Figure 3 to the left an imported mesh in STL file format is shown: STL only supports one single mesh, no visual attributes and no materials: the various building blocks are clearly visible, but cannot be programatically distinguished. To the right we show the result of a engineering round-trip of a hypothetical vertex detector: the ROOT geometry was first exported to a Collada file and subsequently reimported as tessellated shapes in ROOT. Visual attributes and material identifiers are preserved.
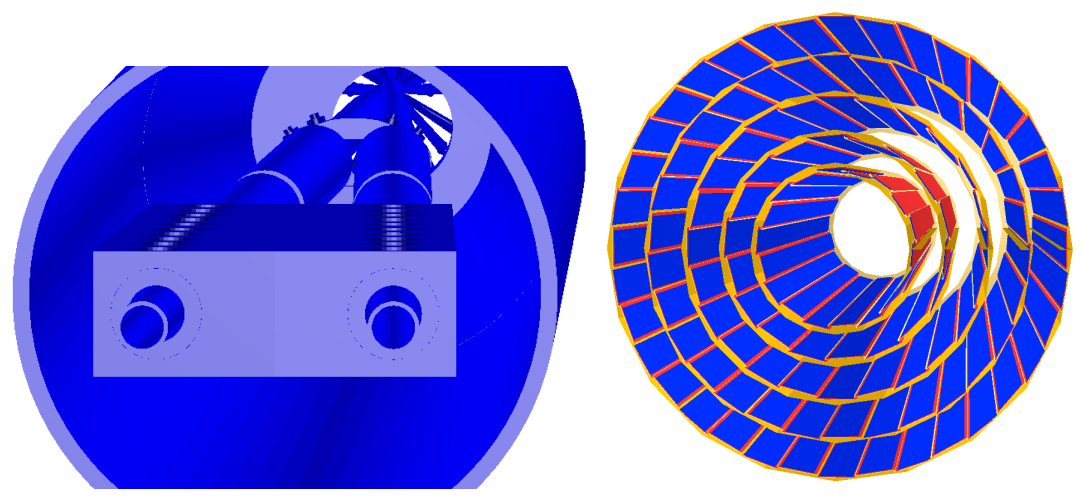

Figure 3. To the left an imported mesh from the STL file format is shown. To the right the result of a round-trip with an intermediate COLLADA file of a hypothetical vertex detector is shown.

Though the inclusion of shapes defined in CAD files into the ROOT geometry description using DD4hep is at an early stage we strongly believe that the potential of this application is very high.

The interface to the assimp library is encapsulated in the DDCad package. If the library is found during the CMake configuration it will be compiled. Using the CMake flag DD4HEP_LOAD_ASSIMP it is also possible to compile the assimp library on demand. 


\section{Geant4 Units}

For decades, the system of units used in experimental particle physics was based on the three basic units: centimeters, seconds and GigaElectronVolts. ROOT and its geometry description package TGeo [4] adopted this convention. For the LHC era the Geant4 collaboration decided that a basic unit system based on millimeters, nanoseconds and MegaElectronVolts is better suited for the LHC experiments. Most experiments using Geant4 today effectively adopted the Geant 4 convention for all areas of data processing: simulation, reconstruction and data analysis. Hence it would be advantageous for experiments using DD4hep (and in turn ROOT) to describe the geometry to have the same system of units in the application code and the description of the experiment's geometry. The geometry itself is per definition agnostic to units, i.e. all length based quantities simply scale by the appropriate factor (factor 10 when converting $\mathrm{cm}$ based geometries to $\mathrm{mm}$ based geometries). Hence to such scaling issues the ROOT geometry toolkit is agnostic. However, derived material related quantities such as density, interaction length or radiation length are defined by non-linear relationships of the basic unit-system quantities. These derived quantities need to be properly redefined based on the underlying basic units.

To allow users to have the same system of units in the geometry description and the application code we supplied a patch to the ROOT geometry description, which allows to switch the ROOT system of units at run-time. This switch can be enabled in DD4hep if required. To avoid confusion between materials described in ROOT units and materials described in Geant4 units, this switch may only be set once, before any element or material is constructed. No geometry toolkit, neither Geant 4 nor ROOT implement the units as compiler dependent type, but rather as floating point numbers. For this reason any convention cannot be enforced.

To enable Geant4 units in DD4hep, the toolkit must be instructed to use Geant4 units, the CMake option DD4HEP_USE_GEANT4_UNITS must be enabled. Client projects using the CMake configuration file provided by DD4hep automatically inherit the correct compilation settings.

\section{Input/Output Plugin Developments}

The Geant4 simulation using a DD4hep based geometry can be done using the ddsim program [7], which offers a wide range of command line or steering file based configuration options. To further ease the simulation workflow and better integrate with different Monte Carlo Generators or frameworks two new IO modules for DDG4 were implemented.

To support a wider range of MC generators, a new plugin was created, which uses the HepMC3 [13] file reader factory to read all formats supported by HepMC3. The HepMC3 : :GenEvent data is then converted into DDG4's internal representation and forwarded to Geant 4 .

To enable simulations for the Key4hep project [14], an output plugin for the EDM4hep event data model was created. The EDM4hep output plugin converts DD4heps internal information about Monte Carlo particles and hits into the respective output collections of EDM4hep and lets them be written to the output file.

Both plugins are optional and can be enabled with the CMake options DD4HEP_USE_HEPMC3 or DD4HEP_USE_EDM4HEP. If the plugins are enabled one can simply use them with the ddsim executable by using the input- and outputfile options with files with the respective extension. For example, ddsim -inputFiles events.hepmc -outputFile simulated_edm4hep.root Because DD4hep already contains a plugin to write out the information from simulated events in a ROOT file, the string edm4hep before the .root extension is mandatory to identify the correct output plugin. 


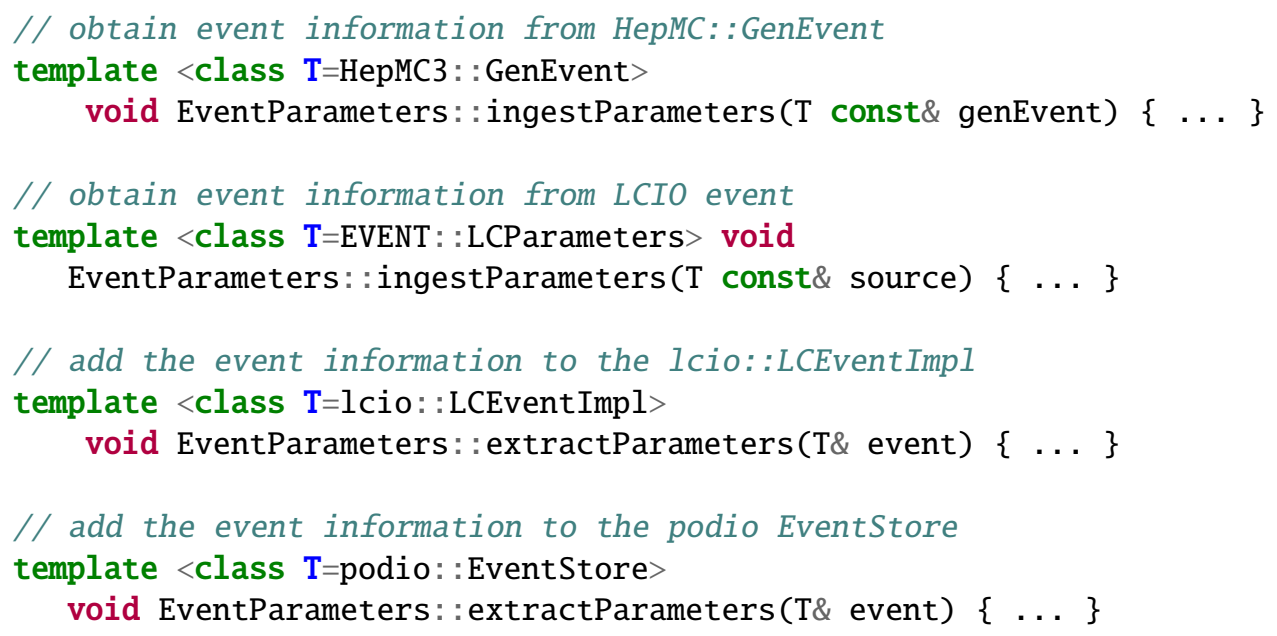

Listing 1: Template instantiation examples for passing event metadata between different inputs and outputs.

To simplify the exchange of meta information between different input and output handlers a dd4hep: : EventParameters class was developed to encapsulate this information. Each input or output module only has only to instantiate and implement a templated function with on the object containing the required information. Listing 1 shows some example signatures of the instantiated functions. The dd4hep: : EventParameters are kept attached to DD4hep's internal event by its extension mechanism.

\section{Conclusion}

The DD4hep Detector Description Toolkit has been providing a fully functional toolset for detector description for many years. The user community of DD4hep is growing steadily, which has triggered a myriad of activities, including new features such as described in these proceedings and, last but not least, also bug fixes. One of the most important new features is the support for tessellated shapes and CAD sources, which adds an important mechanism of importing partial geometries to a comprehensive detector description. Though the inclusion of CAD sources into ROOT geometry description using DD4hep is at a pilot stage, we foresee that the potential for widespread application of these feature is very high. A further improvement in terms of unification of standards was the development of the ability to uniformly use the same system of units throughout DD4hep geometry description as well as in the application code bridging the ROOT vs. Geant4 units gap. It is also noteworthy to mention the extension of the input/output palette that DD4hep supports with the inclusion of broader HepMC support as well as the inclusion of EDM4hep. These developments are a true testimony of the continued strive of the DD4hep developers to improve and evolve DD4hep as a community tool.

\section{Acknowledgements}

This project has received funding from the European Union's Horizon 2020 Research and Innovation programme under Grant Agreement no. 654168. 


\section{References}

[1] M. Frank, F. Gaede, M. Petric, A. Sailer, AIDASoft/DD4hep (2018), webpage: http://dd4hep.cern.ch/, https://doi .org/10.5281/zenodo. 592244

[2] S. Ponce, P. Mato Vila, A. Valassi, I. Belyaev, eConf C0303241, THJT007 (2003), physics $/ 0306089$

[3] LCC, The Linear Collider Collaboration, https://www . linearcollider .org/

[4] R. Brun, A. Gheata, M. Gheata, Nucl. Instrum. Meth. A502, 676 (2003)

[5] S. Agostinelli et al. (GEANT4), Nucl. Instrum. Meth. A506, 250 (2003)

[6] M. Frank, F. Gaede, C. Grefe, P. Mato, J. Phys. Conf. Ser. 513, 022010 (2014)

[7] M. Petrič, M. Frank, F. Gaede, S. Lu, N. Nikiforou, A. Sailer, Journal of Physics: Conference Series 898, 042015 (2017)

[8] F. Gaede, M. Frank, M. Petric, A. Sailer, EPJ Web Conf. 245, 02004 (2020)

[9] J.A. et al., IEEE TNS, 53 1, 270 (2006)

[10] K. Kulling, Open asset import library (assimp), http: //www . assimp .org

[11] M. Frank, F. Gaede, N. Nikiforou, M. Petric, A. Sailer, J. Phys. Conf. Ser. 664, 072017 (2015)

[12] K. Kulling, Open asset import library (assimp), source code, https://github.com/ assimp/assimp

[13] A. Buckley, P. Ilten, D. Konstantinov, L. Lönnblad, J. Monk, W. Pokorski, T. Przedzinski, A. Verbytskyi, Comput. Phys. Commun. 260, 107310 (2021), 1912.08005

[14] A. Sailer, G. Ganis, P. Mato, M. Petrič, G.A. Stewart, EPJ Web Conf. 245, 10002 (2020) 